\title{
Ultraviolet to far-infrared transmission properties of thin film multi-walled carbon nanotube random networks
}

\author{
M. Wąsik ${ }^{1, \star}$, A. Dużyńska ${ }^{1}$, J. Judek $^{1}$, M. Pawłowski ${ }^{1}$, K. Świtkowski $^{1}$, A. M. Witowski ${ }^{2}$, and M. Zdrojek ${ }^{1}$ \\ ${ }^{1}$ Faculty of Physics, Warsaw University of Technology, Koszykowa 75, 00-662 Warsaw, Poland \\ ${ }^{2}$ Faculty of Physics, Institute of Experimental Physics, University of Warsaw, Pasteura 5, 02-093 Warsaw, Poland
}

Received: 24 July 2016

Accepted: 14 November 2016

Published online:

28 November 2016

(C) The Author(s) 2016. This article is published with open access at Springerlink.com

\begin{abstract}
Thin films of multi-walled carbon nanotubes forming random networks were produced by vacuum filtration method, and their broadband electromagnetic radiation transmittance spectra are presented. Thickness of the nanotube films was between $100 \mathrm{~nm}$ and $1 \mu \mathrm{m}$, and the transmission properties are demonstrated for the wavelength range from $300 \mathrm{~nm}$ to $400 \mu \mathrm{m}$. It is observed that transmittance is an increasing function of a radiation wavelength, and for the thickest films it almost saturates above $1 \mu \mathrm{m}$ wavelength. To explain the experimental results in the ultraviolet-near infrared range, we employed effective medium theory (in the form of symmetric Bruggeman model) correlating properties of multi-walled carbon nanotubes with the effective dielectric function of a nanotube network. The optical properties of a single multi-walled carbon nanotube that were used for calculations were based on ordinary and extraordinary dielectric functions of bulk graphite. The proposed theoretical model has been successfully fitted to the experimental results. It has been also found that despite the fact that radiation undergoes multiple internal reflections at the film interfaces, the transmittance-thickness relation can be still described by exponential decay.
\end{abstract}

\section{Introduction}

The interaction between electromagnetic radiation and carbon nanotubes has been continually the subject of scientific interest, both from the theoretical and experimental point of view. In case of single-walled carbon nanotubes, the characteristic features of their optical spectra can be correlated with theoretical interband transitions and are very sensitive to chiral indices describing a nanotube [1, 2]. For multi-walled carbon nanotubes (MWCNTs) with diameters larger than few nanometers, the curvature effects can be neglected and optical properties of a single MWCNT can be derived from the dielectric functions of bulk graphite [3]. The anisotropy of both a nanotube (high aspect ratio) and graphite properties is the reason

Address correspondence to E-mail: wasik@if.pw.edu.pl 
why the overall way carbon nanotubes interact with electromagnetic waves depending on their specific arrangement in an investigated sample. The experimental or theoretical analyses of this issue were addressed for single MWCNT [4], their bundles [5], and very extensively for vertically aligned nanotube arrays [6-9]. A widely discussed topic is electromagnetic waves shielding potential of composites where MWCNTs are inclusion responsible for energy dissipation [10, 11].

As far as optical properties of thin films of the pure carbon nanotubes are concerned, the studies mainly concentrate on single-walled carbon nanotubes with clearly insufficient information about MWCNTs [12]. For instance, the absorbance spectrum for layers of MWCNTs grown in alumina templates was reported in limited, mid infrared range $(1.5-10 \mu \mathrm{m})$ [13], and the transmission-reflection-transmission configuration used is not straightforward to interpret. The wider spectral range extending to far-infrared (up to $\sim 167 \mu \mathrm{m}$ ) was presented by Kamarás et al. [14] but neither visible nor UV part of electromagnetic spectrum was presented. In the work of De Nicola et al. [15], transmittance spectra for films of single- and multi-walled nanotubes are compared and are limited to the Vis-NIR range. Only three thickness values are used there to estimate the absorption coefficient with no substrate influence analysis included. What is more, there are no reports that would demonstrate the application of effective medium theory to explain the transmittance spectra of pure MWCNT thin films forming random networks. In the presented study, the consistent and broad (from UV to far-infrared) range of electromagnetic spectrum is covered and the results are confronted with the effective medium approach. It should be emphasized that the knowledge about such fundamental quantities as transmittance is a key factor when designing of MWCNT-based optical materials (and also optoelectronic devices) is considered. The effective operation of such systems for radiation from a wide frequency range makes the given material more attractive from the application point of view. Despite tendencies toward studies on hybrid composites with nanotubes (often based on polymer matrix and other nanoparticles), it is clear that having the detailed picture of the MWCNTs transmission properties is a reasonable starting point for analysis of more complex systems based on carbon nanotubes.
The films of MWCNT can be prepared with methods such as drop casting, spin/spray coating, electrophoresis deposition, or vacuum filtration [16]. Despite macroscopic uniformity of such samples, the internal structure of nanotube films can be very complex and formally they should be treated as the combination of a host material (most often air) and carbon inclusions. For a theoretical description of electromagnetic properties of such materials, the literature delivers different models being classified under effective medium theory (EMT) and so far they were mainly applied to systems of vertically aligned nanotubes [17-19]. The theory concentrates on predicting the effective dielectric function $\varepsilon_{\mathrm{eff}}$ of a material that consists of the clearly distinguishable components, taking into account the properties of all phases. In general, the most popular EMT approaches are Maxwell-Garnett, Bruggeman, and the socalled power laws (including, e.g., Birchak, Drude, or Looyenga formulas) [20, 21]. The first one has been successfully used for simulations of reflectance and absorbance spectra of carbon nanotube forests in different configurations [8, 22]. Apart from the homogenization procedure (finding $\varepsilon_{\text {eff }}$ ), for thin film samples, the analysis of transmission properties of the material itself requires considerations of multiple reflections at the interfaces which makes the theoretical expressions describing optical spectra much more complex [23]. In our work, we adopted the Bruggeman mixing formula to model the effective optical properties of MWCNT random networks and considered them as a part of four layer system: air/ MWCNTs/substrate/air. For demonstration of transmittance spectra $T(\lambda)$ in the broadband range, from ultraviolet to far-infrared (300 $\mathrm{nm}$ to $400 \mu \mathrm{m}$ ), a sample without substrate was prepared and studied.

\section{Experimental details}

\section{Preparation of MWCNT films}

The MWCNT films were fabricated by the following procedure. For preparation of a nanotubes solution, dry powder of MWCNTs from NanoIntegris (purity $99 \%$, tube length 1-12 $\mu \mathrm{m}$, inside diameter $4 \mathrm{~nm}$, outer diameter $<20 \mathrm{~nm}$ ) was diluted in $1 \%$ water solution of sodium dodecyl sulfate to achieve a $0.2 \mathrm{mg} / \mathrm{mL}$ nanotube concentration. Dispersion was placed in a sonic bath for $3 \mathrm{~h}$ (in cool water) and then centrifuged at 
Figure 1 Pictures of the investigated MWCNT films. a, b The scanning electron microscope images. $\mathbf{c}$ The real samples on glass substrate. d A scheme of the sample supported on a metal substrate with a hole.
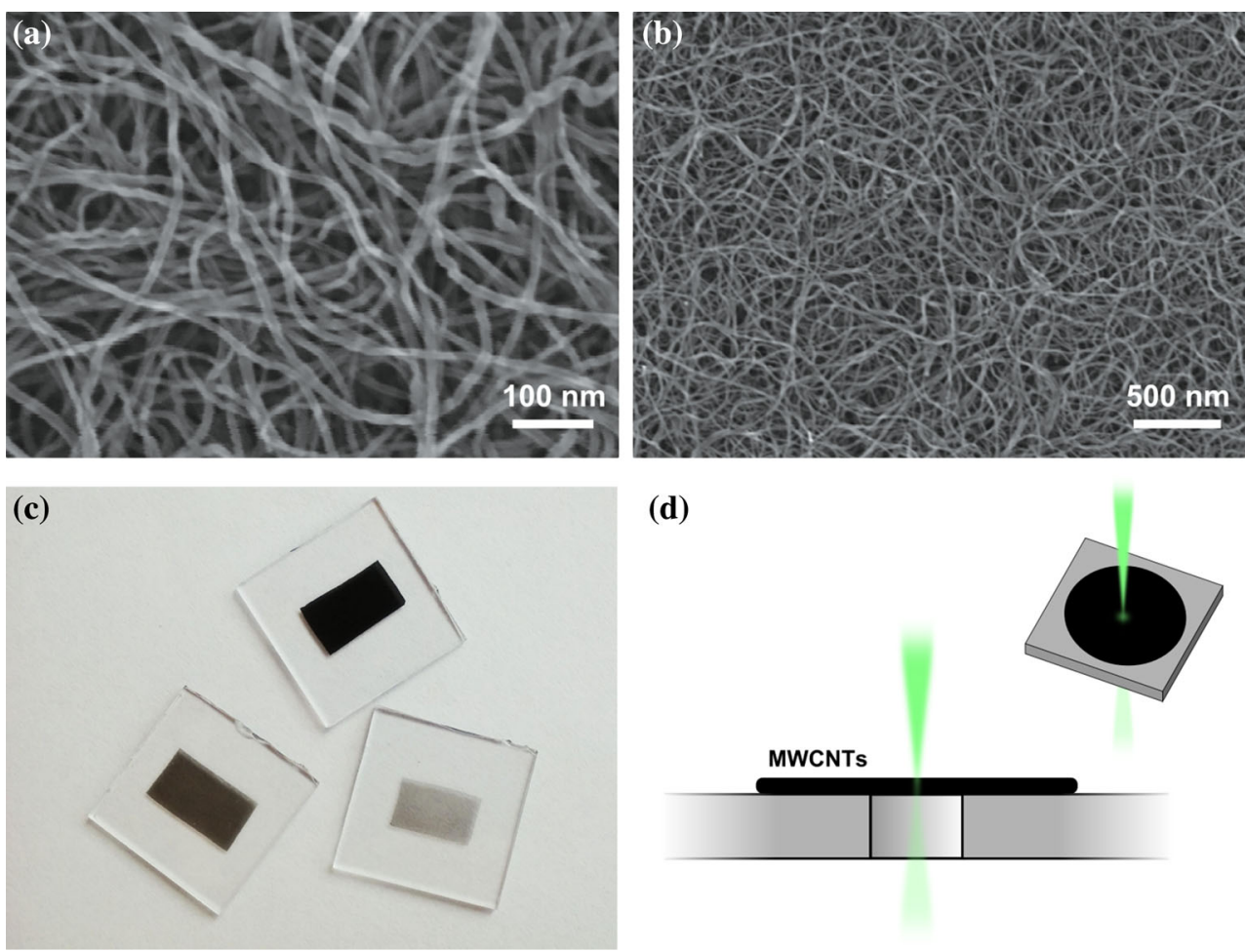

(d)

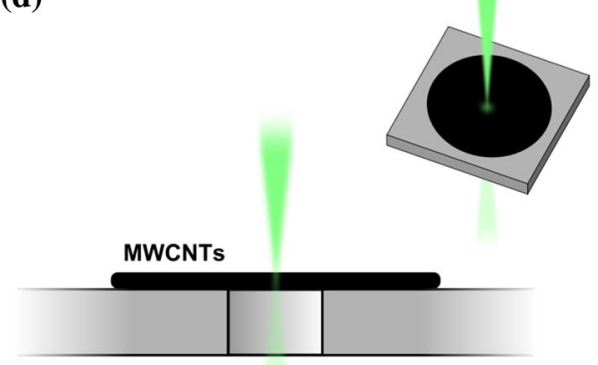

$15,000 \mathrm{rpm}$ for $10 \mathrm{~min}$ to separate the homogeneous solution from bundles of MWCNTs. The supernatant was carefully decanted and saved. Based on as-prepared solution, the thin films were fabricated by a vacuum filtration method [24] using mixed cellulose ester membranes $(0.025 \mu \mathrm{m}$ pore size, $25 \mathrm{~mm}$ diameter) from Millipore. Thicknesses of carbon nanotube films were controlled by the volume of the filtered solution along with atomic force microscope images (NT-MDT, semi-contact mode). The prepared films were cut and transferred to the soda lime glass plates (Fig. 1c). Six produced films on glass substrate were investigated and their thicknesses were approximately equal to 100, 185, 230, $450 \mathrm{~nm}, 0.86$, and $1 \mu \mathrm{m}$. The values were obtained from atomic force microscope scans performed at different regions along the layer/substrate boundary, and the final value is a result of averaging procedure. An additional MWCNT layer was placed on a metal substrate with a hole of $3 \mathrm{~mm}$ in diameter (Fig. 1d) so that the optical spectra undisturbed by the presence of a substrate could be measured in the broad wavelength range.

\section{Characterization of MWCNT films}

The internal structure of the obtained thin films is similar to a network of nanotubes tending to arrange in a layered structure rather than chaotic bundles. This can be deduced from the scanning electron microscope images of the MWCNT networks shown in Fig. 1a, b. To estimate the volume filling factor of the discussed samples, a thick test MWCNT layer was prepared and based on measurements of its weight and physical dimensions, the density of a layer was determined. A comparison of this value to an averaged tube density gives the estimated filling factor value of $\sim 0.2$. The transmittance spectra (at normal incidence angle) for wavelengths from $300 \mathrm{~nm}$ to $2 \mu \mathrm{m}$ were collected with use of an Agilent Technologies Cary 5000 spectrometer and for the spectral range between 2 and $400 \mu \mathrm{m}$ with a Bruker $113 \mathrm{v}$ vacuum Fourier transform spectrometer. All measurements were carried out at room temperature.

\section{Results and discussion}

\section{Transmittance spectra}

The relative transmittance spectra $T_{\text {rel }}(\lambda)$ of MWCNT thin films collected for six different samples on glass substrates are presented in Fig. 2, and they are calculated with respect to the substrate spectrum $T_{\text {sub }}(\lambda)$ so that they better demonstrate the properties of 


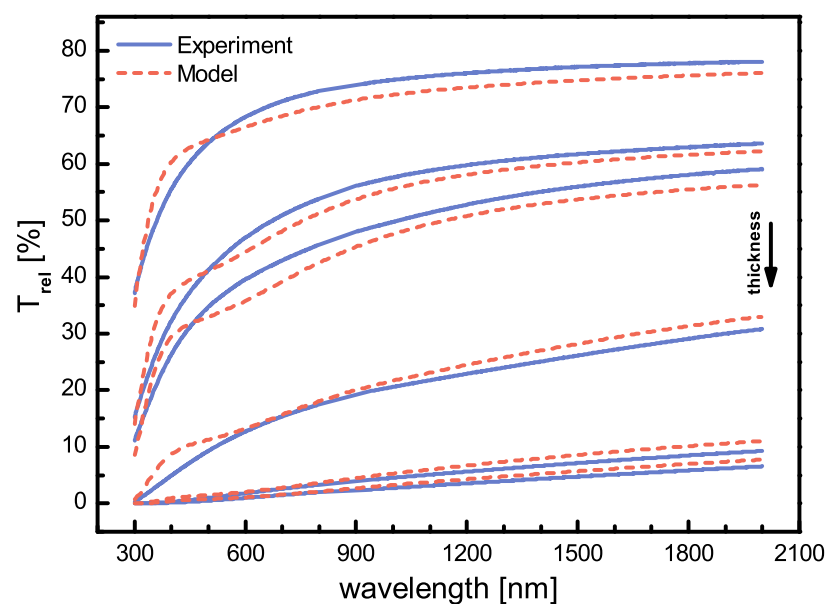

Figure 2 The relative transmittance spectra of the MWCNT films of different thicknesses (given in the text) on glass substrate. Solid lines experimental values, dashed lines values obtained from EMT model.

material itself. It is clearly seen that the thicker a MWCNT film is, the faster the transmission signal is saturated (flattened). In order to obtain $T(\lambda)$ for midand far-infrared range, the MWCNT layer partially suspended in air was used (of thickness $\sim 0.77 \mu \mathrm{m}$ ), and the Fourier spectrometer was employed. The total spectrum for this sample covering the spectral range from UV to far-infrared is shown in Fig. 3. It can be noticed that the general tendency observed for transmittance curves quantitatively agrees with the data demonstrated by Kamarás et al. [14] for free standing layers of MWCNTs.

\section{Effective medium theory model}

To explain the experimental results in UV-Vis-NIR spectral region, we used the effective medium theory in a form of the Bruggeman symmetric Eq. (1) relating the dielectric functions of $N$ constituent materials/inclusions characterized by $\varepsilon_{i}$ (each occupying $f_{\mathrm{i}}$ volume fraction) to the effective dielectric function of a new homogenous medium $\varepsilon_{\text {eff }}$.

$\sum_{i=1}^{N} f_{i} \frac{\varepsilon_{i}-\varepsilon_{\mathrm{eff}}}{\varepsilon_{\mathrm{eff}}+L\left(\varepsilon_{i}-\varepsilon_{\mathrm{eff}}\right)}=0, \quad \sum_{i=1}^{N} f_{i}=1$.

The parameter $L$ is a so-called depolarization factor and can be strictly calculated if the shape of a single inclusion is known. The choice of the Bruggeman approach is justified by the fact that it is generally considered as giving more reliable results if filling factors are not very small which is the case for the presented samples [25].

The first step toward obtaining $\varepsilon_{\text {eff }}$ is a decomposition of the nanotube network into two subsets of nanotubes (or rather their segments)-MWCNTs perpendicular and parallel to an arbitrary direction on a plane of the film. Such division is dictated by the fact that two different dielectric functions describe interactions between electromagnetic radiation and a

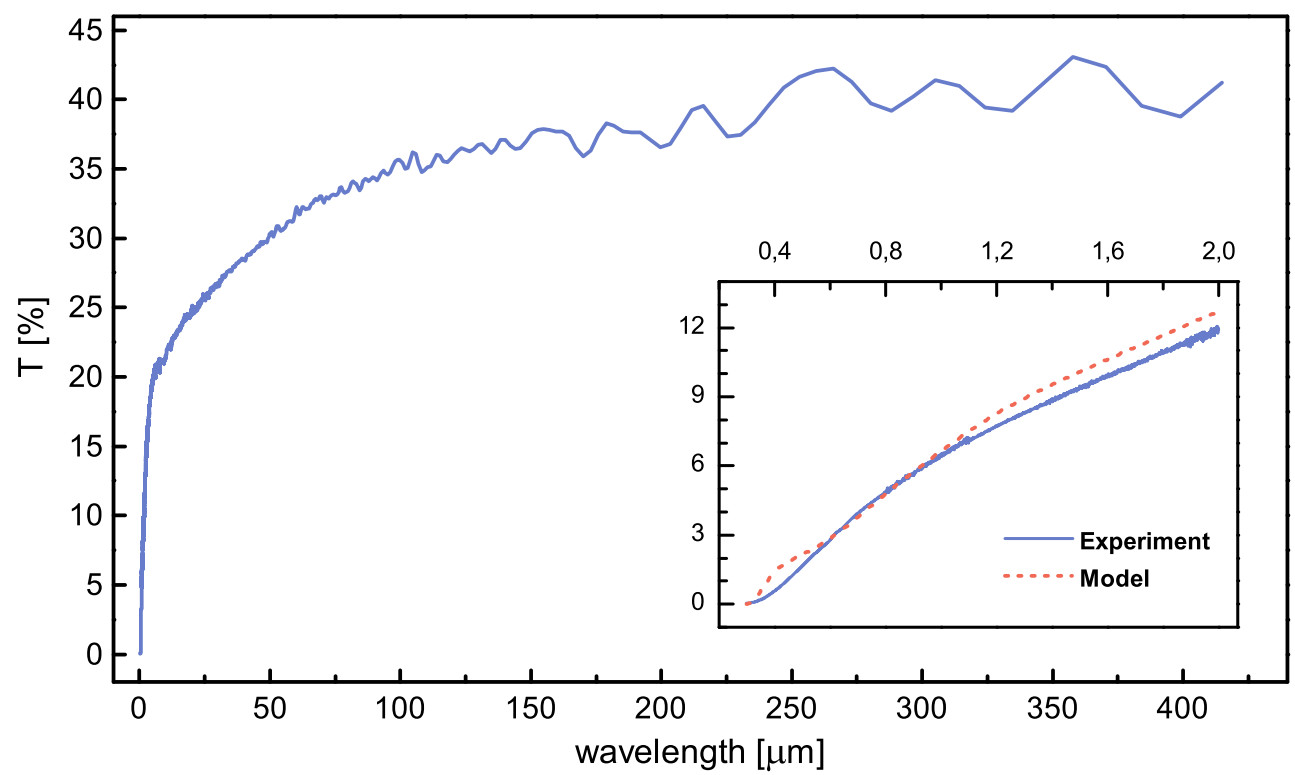

Figure 3 Transmittance spectrum of the MWCNT film with no substrate. The oscillations in far-infrared come from measurement noise. The inset shows the UV-Vis-NIR region with solid (dashed) curve corresponding to the experimental (EMT model) results. 


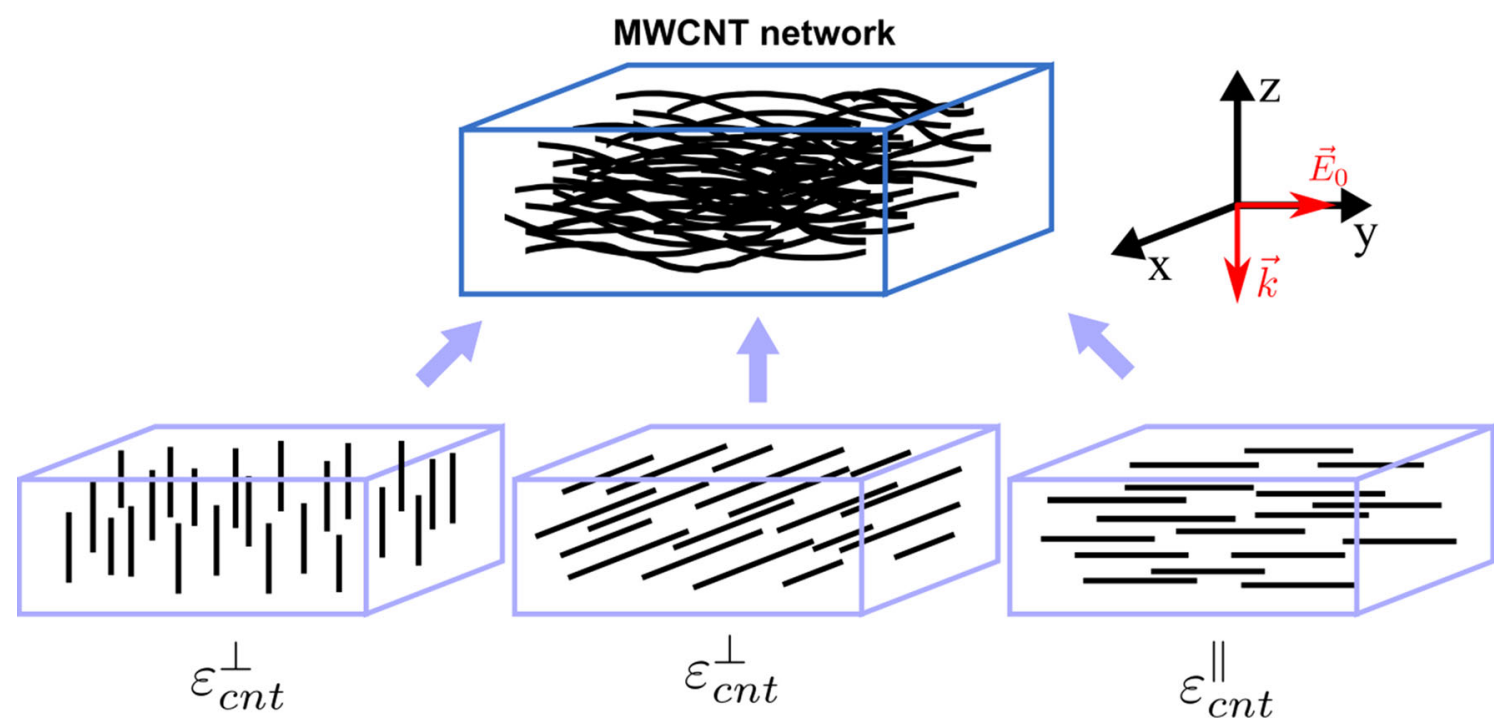

Figure 4 Schematic decomposition of MWCNT network into components assigned to two dielectric functions $\varepsilon_{\mathrm{CNT}}^{\|}$and $\varepsilon_{\mathrm{CNT}}^{\stackrel{\perp}{ }}$. The surface of the layer lies within $x-y$ plane. The direction and

nanotube, depending on a mutual orientation of a polarization vector of incoming radiation and the tube axis. This situation was schematically depicted in Fig. 4. In both cases, we consider the two-component mixture of the MWCNT inclusions-of the same filling factor $f_{0}$-described by functions $\varepsilon_{\mathrm{CNT}}^{\|}$(parallel configuration) and $\varepsilon_{\mathrm{CNT}}{ }^{\perp}$ (perpendicular configuration) with air at volume fraction of $1-f_{0}$. Obviously, the concentration of nanotube segments of perpendicular or parallel configuration in the real network is not equal to $f_{0}$ (which is the overall concentration) but this fact is to be taken into account later. It should be also noticed that two different depolarization factors are used here, denoted as $L_{1}$ and $L_{2}$ for parallel and perpendicular configurations, respectively. As a consequence, Eq. (1) is solved twice (for $\varepsilon_{\text {eff }}$ " and $\varepsilon_{\text {eff }}{ }^{\perp}$ ) and takes a quadratic form where the right solution is chosen so that it satisfies the natural condition: $\varepsilon_{\mathrm{eff}}^{\| / \perp}\left(f_{o} \rightarrow 0\right)=\varepsilon_{\text {air }}=1$ The functions $\varepsilon_{\mathrm{CNT}} \|$ and $\varepsilon_{\mathrm{CNT}}{ }^{\perp}$ are adopted as presented in $[3,26]$ and they are derived based on the literature data available for ordinary and extraordinary dielectric functions of bulk graphite. The limitations in spectral range for these datasets are the reason why the proposed EMT model is not extended to mid- and far-infrared range.

The second stage for determination of the final $\varepsilon_{\text {eff }}$ is combining the solutions $\varepsilon_{\mathrm{eff}}^{\|}$and $\varepsilon_{\mathrm{eff}}^{\perp}$ to account for random distribution of carbon nanotubes within the network. In the proposed model, we assumed the polarization state of incoming radiation are determined by vectors $\vec{k}$ and $\vec{E}_{0}$, respectively.

linear combination given by Eq. (2), as suggested by e.g., $[6,9]$, with normalized weights.

$\varepsilon_{\mathrm{eff}}=(1-p) \varepsilon_{\mathrm{eff}}^{\|}+p \varepsilon_{\mathrm{eff}}^{\perp}$.

The random distribution of nanotubes in a plane of the film may suggest that the weights $(1-p)$ and $p$ should be both equal to 0.5 ; however, the fact of nanotubes bending in a direction perpendicular to the layer plane should be expressed in $p$ values being higher than 0.5 (see also Fig. 4, first component).

Once $\varepsilon_{\text {eff }}$ is evaluated for different wavelengths, one can use it to calculate the transmittance spectra of air/MWCNTs/air and air/MWCNTs/substrate/air systems. In the first case (as in Fig. 1d), the expression for the film spectral transmittance at normal incidence $T_{\text {film }}$, taking into account multiple reflections at both interfaces (Fabry-Pérot effect), is given by Eq. (3) [23]:

$T_{\text {film }}=\left|\frac{\left(1-r_{\mathrm{a}-\mathrm{f}}^{2}\right) \exp (-2 \pi \mathrm{ind} / \lambda)}{1-r_{\mathrm{a}-\mathrm{f}}^{2} \exp (-2 \pi \mathrm{ind} / \lambda)}\right|^{2}$.

In the formula (3), $n$ is a real part of the complex refractive index $\tilde{n}_{\text {eff }}(\lambda)=n_{\text {eff }}(\lambda)+i k_{\text {eff }}(\lambda)$ related to the effective dielectric function $\left[\varepsilon_{\mathrm{eff}}(\lambda)=\tilde{n}_{\mathrm{eff}}^{2}(\lambda)\right] ; r_{\mathrm{a}-\mathrm{f}}$ is the Fresnel reflection coefficient (being function of $\tilde{n}_{\text {eff }}$ ) for radiation coming from air into the film; and $d$ is a thickness of the film. For analysis of transmittance spectra of films on glass substrate, much more complex formula can be derived that includes the 
effect of multiple reflections at three interfaces. For our calculations, we used relations given in [23] with the additional assumption that the thickness of a substrate is much larger than an incoming radiation wavelength which is valid in our case.

\section{EMT fitting results}

Following the outlined procedures, we arrive at the full model for the transmittance spectra for the MWCNT random network films with four parameters $\left(f_{0}, L_{1}, L_{2}, p\right)$ that can be varied within different constraints so that the best fit to the experimental results is obtained. The filling factor $f_{0}$ was experimentally estimated to be equal to 0.2 with uncertainty of $\sim 20 \%$, and the expected value for $p$ was assumed to lie between $1 / 2$ and $2 / 3$ which corresponds to two limiting cases of tubes arrangement in the layer (perfect layer-by-layer and chaotic bundles). As far as depolarization factors are concerned, an arbitrary ellipsoid particle can be characterized by three depolarization factors $\left(L_{\mathrm{x}}, L_{\mathrm{y}}, L_{\mathrm{z}}\right)$ whose sum is equal to 1 [27]. The depolarization factors for a very long and thin cylinder (a needle) are equal to $(0.5,0.5,0)$ [28] and can serve as initial guesses for the values describing a MWCNT. It can be expected that both tube-tube interactions and their bending are supposed to affect these values [29]. As in the case of a long needle we assumed that for the single MWCNT in the network, the effective values of $L_{\mathrm{x}}$ and $L_{\mathrm{y}}$ are equal (denoted as $L_{2}$ ), and the sum of all depolarization factors is 1 . This leads to the condition $L_{\mathrm{z}} \equiv L_{1}=0.5\left(1-L_{2}\right)$ which is used to reduce the number of degrees of freedom in the model.

The fitting procedure with the above formulated constraints yields the values $f_{0}=0.220, p=0.583$, $L_{1}=0.142, L_{2}=0.431$, and the predicted transmittance curves in the range of $(0.3-2) \mu \mathrm{m}$ are presented in Fig. 2 and in the inset of Fig. 3. The modeled transmittance values $\left(T^{\prime}\right)$ for films on a substrate are calculated with respect to the glass substrate transmittance $\left(T^{\prime}=T / T_{\text {sub }}\right)$ so that both experimental and theoretical results are presented in the same way. The matching between fitted spectra and experimental data is satisfying (the discrepancies do not exceed 5\% in an absolute scale) given the limitations of effective medium approximation. For example, the general assumption beyond discussed EMT is that the characteristic dimensions of inclusions are supposed to be much smaller than radiation wavelength. With respect to
MWCNTs diameter, the condition is satisfied. However, when dimensions of local irregular sub-micrometer structures formed by MWCNTs are compared to $\lambda$, the assumption may no longer be valid. Also, one has to keep in mind that EMT is usually applied to systems of spatially noninteracting inclusions. The proposed theory accounts for the details of relatively complicated geometry of the nanotube network by treating (within physical restrictions) the depolarization factor as a fitting parameter but possible wavelength-dependent scattering effects are still ignored. Because addressing these issues would lead to much increase in the complexity of our model $[30,31]$, so taking into account the good agreement between measurements results and fitted values, the presented approach can be viewed as suitable for handling the discussed materials.

Based on the presented model, we also calculated the relative transmittance curves $T^{\prime}$ as a function of a MWCNT layer thickness and compared them to exponential decays $\exp \left(-\alpha_{\mathrm{eff}} d\right)$ characterized by corresponding effective absorption coefficient $\alpha_{\text {eff }}$ calculated with EMT as well. The comparison shown in Fig. 5 for three different wavelengths $(0.5,1$, and $2 \mu \mathrm{m})$ demonstrates that both types of curves differ only slightly. This indicates that the procedure of dividing full $T$ values by substrate transmittance efficiently accounts for reflection effects within air/MWCNT/ substrate/air system. It is then quite natural that experimentally fitted absorption coefficient agrees

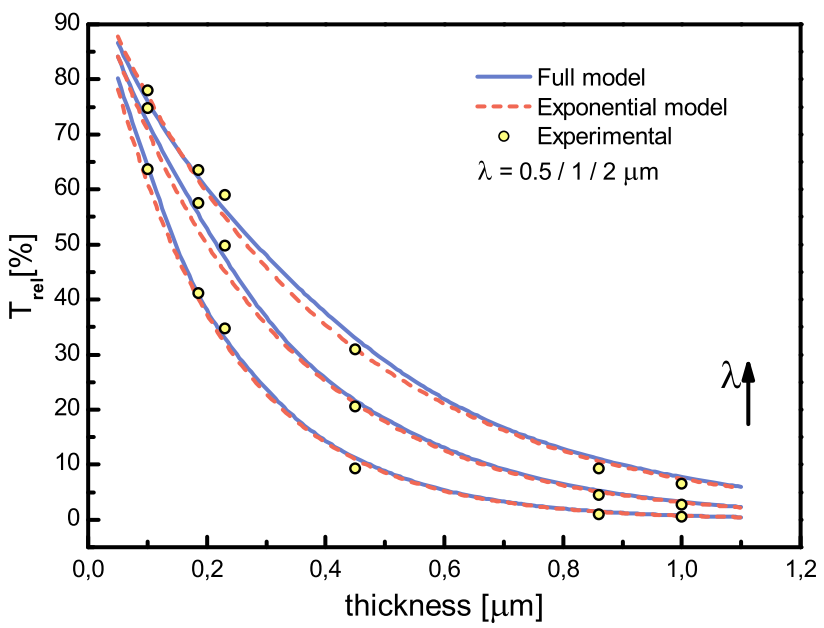

Figure 5 Relative transmittance of the MWCNT films on glass substrates for three wavelengths $(0.5,1$, and $2 \mu \mathrm{m})$ as a function of the film thickness. Solid curves correspond to direct calculations of transmission signal and dashed curves present exponential decays $\exp \left(\alpha_{\mathrm{eff}} d\right)$. Circles represent experimental values. 


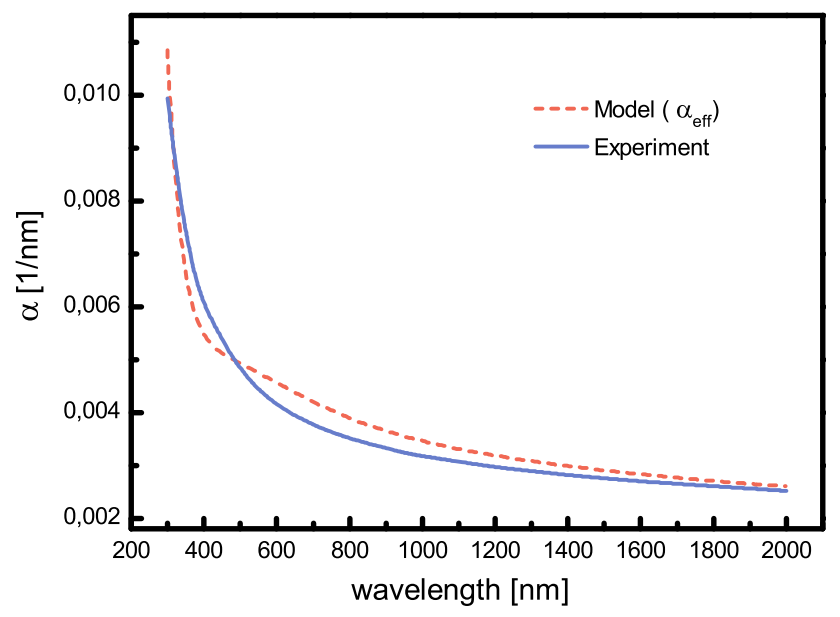

Figure 6 Absorption coefficient of MWCNT film as a function of wavelength calculated from EMT model (dashed curve) and extracted from the experimental results by fitting-at each wavelength step - to exponent (solid curve).

with the function $\alpha_{\text {eff }}(\lambda)$ obtained from EMT model, as shown in Fig. 6. The importance of this conclusion is the fact that the amount of radiation blocked by the MWCNT layer of arbitrary thickness can be therefore described with the use of only one parameter. It is the significant simplification in estimating shielding efficiency when there is no possibility of preparing elaborate samples of films suspended in air or free standing, as it is in case of very thin films.

It should be noted that the above result is valid for layers on glass substrate and may not be applicable in case of substrates of other optical properties especially those of high reflection coefficient. As a final remark it is worth highlighting that contrary to UV region, radiation shielding potential of MWCNT films in the infrared is significantly reduced. Interestingly, in case of single-walled carbon nanotube films, the reported far-infrared behavior of transmission signal is different $[32,33]$, with a strong absorption peak centered usually at $\sim 100 \mu \mathrm{m}$. This structure may be observed in MWCNTs but only for tubes with high enough crystallinity level [34]. In our case this condition is not satisfied as some bends and kinks in tubes structure are noticeable in the electron microscope pictures.

\section{Conclusions}

By investigating the broadband transmittance spectra of the thin films of MWCNT random networks, we concluded that their transmittance monotonically decreases as wavelength is increased and the kind of saturation is observed that depends on the sample thickness. The experimental data in the visible to near infrared range were successfully fitted with the theoretical spectra calculated based on effective medium theory formulated in the Bruggeman approach. Further improvements to the model, extending beyond EMT, would presumably require the detailed analysis of scattering events within complex structures formed by single MWCNTs. The proposed model fully incorporates the details of radiation propagation in an effective medium including the influence of a substrate. It turns out that the transmittance spectra calculated relative to the glass substrate transmittance approximately follow an exponential decay with respect to the sample thickness which allows for the straightforward estimation of the shielding potential of MWCNT random network films.

\section{Acknowledgements}

This research was partially funded under Grant DI 2011029441 of Polish Ministry of Science and Higher Education and also under the Project Preludium of the National Science Centre (2014/13/N/ST5/01255).

\section{Compliance with ethical standards}

Conflict of interest The authors declare that there is no conflict of interest.

Open Access This article is distributed under the terms of the Creative Commons Attribution 4.0 International License (http://creativecommons.org/licen ses/by/4.0/), which permits unrestricted use, distribution, and reproduction in any medium, provided you give appropriate credit to the original author(s) and the source, provide a link to the Creative Commons license, and indicate if changes were made.

\section{References}

[1] Bachilo SM, Strano MS, Kittrell C, Hauge RH, Smalley RE, Weisman RB (2002) Structure-assigned optical spectra of single-walled carbon nanotubes. Science 298(5602):2361-2366

[2] Hagen A, Hertel T (2003) Quantitative analysis of optical spectra from individual single-wall carbon nanotubes. Nano Lett 3(3):383-388

[3] Shamsollahi Y, Moravvej-Farshi MK, Ebnali-Heidari M (2013) Photonic crystals based on periodic arrays of 
MWCNTs: modeling and simulation. J Lightwave Technol 31(12):1946-1953

[4] Zdrojek M, Judek J, Wąsik (2012) Laser heating control with polarized light in isolated multiwalled carbon nanotubes. Phys Rev Lett 108(22):225501

[5] Lin MF, Shyu FL, Chen RB (2000) Optical properties of well-aligned multiwalled carbon nanotube bundles. Phys Rev B 61(20):14114-14118

[6] Wood BD, Dyer JS, Thurgood VA, Tomlin NA, Lehman JH, Shen TC (2015) Optical reflection and absorption of carbon nanotube forest films on substrates. J Appl Phys 118(1):013106

[7] Zhang RZ, Liu X, Zhang ZM (2015) Modeling the optical and radiative properties of vertically aligned carbon nanotubes in the infrared region. J Heat Transf 137(9):091009. doi: $10.1115 / 1.4030222$

[8] Ye H, Wang XJ, Lin W, Wong CP, Zhang ZM (2012) Infrared absorption coefficients of vertically aligned carbon nanotube films. Appl Phys Lett 101(14):141909. doi:10. $1063 / 1.4757395$

[9] Wang XJ, Flicker JD, Lee BJ, Ready WJ, Zhang ZM (2009) Visible and near-infrared radiative properties of vertically aligned multi-walled carbon nanotubes. Nanotechnology 20(21):215704. doi:10.1088/0957-4484/20/21/215704

[10] Fan Z, Luo G, Zhang Z, Zhou L, Wei F (2006) Electromagnetic and microwave absorbing properties of multiwalled carbon nanotubes/polymer composites. Mater Sci Eng B 132(1-2):85-89

[11] Al-Saleh MH, Sundararaj U (2009) Electromagnetic interference shielding mechanisms of CNT/polymer composites. Carbon 47(7):1738-1746

[12] Lehman JH, Terrones M, Mansfield E, Hurst KE, Meunier V (2011) Evaluating the characteristics of multiwall carbon nanotubes. Carbon 49(8):2581-2602

[13] Kouklin N, Tzolov M, Straus D, Yin A, Xu JM (2004) Infrared absorption properties of carbon nanotubes synthesized by chemical vapor deposition. Appl Phys Lett 85(19):4463-4465

[14] Kamarás K, Pekker Á, Bruckner M, Borondics F, Rinzler AG, Tanner DB, Itkis ME, Haddon RC, Tan Y, Resasco DE (2008) Wide-range optical spectra of carbon nanotubes: a comparative study. Phys Status Solidi B 245(10):2229-2232

[15] De Nicola F, Pintossi C, Nanni F, Cacciotti I, Scarselli M, Drera G, Pagliara S, Sangaletti L, De Crescenzi M, Castrucci $P$ (2015) Controlling the thickness of carbon nanotube random network films by the estimation of the absorption coefficient. Carbon 95:28-33

[16] Kanoun O, Müller C, Benchirouf A, Sanli A, Dinh TN, AlHamry A, Bu L, Gerlach C, Bouhamed A (2014) Flexible carbon nanotube films for high performance strain sensors. Sensors 14(6):10042-10071
[17] Lü W, Dong J, Li ZY (2001) Optical properties of aligned carbon nanotube systems studied by the effective-medium approximation method. Phys Rev B 63(3):334011-334014

[18] García-Vidal FJ, Pitarke JM, Pendry JB (1997) Effective medium theory of the optical properties of aligned carbon nanotubes. Phys Rev Lett 78(22):4289-4292

[19] Wu XH, Pan LS, Fan XJ, Xu D, Li H, Zhang CX (2003) A semi-analytic method for studying optical properties of aligned carbon nanotubes. Nanotechnology 14(11):11801186

[20] Goncharenko AV, Lozovski VZ, Venger EF (2000) Lichtenecker's equation: applicability and limitations. Opt Commun 174(1-4):19-32

[21] Hutchinson NJ, Coquil T, Richman EK, Tolbert SH, Pilon L (2010) Reflectance of surfactant-templated mesoporous silica thin films: simulations versus experiments. Thin Solid Films 518(8):2134-2140

[22] Mukherjee S, Misra A (2014) Broadband wavelength-selective reflectance and selective polarization by a tip-bent vertically aligned multi-walled carbon nanotube forest. J Phys D 47(23):235501

[23] Chen JJ, Lin JD, Sheu LJ (1999) Simultaneous measurement of spectral optical properties and thickness of an absorbing thin film on a substrate. Thin Solid Films 354(1):176-186

[24] Wu Z, Chen Z, Du X, Logan JM, Sippel J, Nikolou M, Kamaras K, Reynolds JR, Tanner DB, Hebard AF, Rinzler AG (2004) Transparent, conductive carbon nanotube films. Science 305(5688):1273-1276

[25] Markel VA (2016) Introduction to the Maxwell Garnett approximation: tutorial. J Opt Soc Am A 33(7):1244-1256

[26] Djurišić AB, Li EH (1999) Optical properties of graphite. J Appl Phys 85(10):7404-7410

[27] Giordano S (2003) Effective medium theory for dispersions of dielectric ellipsoids. J Electrost 58(1-2):59-76

[28] Sihvola A (2007) Dielectric polarization and particle shape effects. J Nanomater. 2007(2007):45090. doi:10.1155/2007/ 45090

[29] Fanchini G, Unalan HE, Chhowalla M (2006) Optoelectronic properties of transparent and conducting single-wall carbon nanotube thin films. Appl Phys Lett 88(19):191919. doi:10.1063/1.2202703

[30] Mikki SM, Kishk AA (2007) Theory of optical scattering by carbon nanotubes. Microw Opt Technol Lett 49(10):2360 2364

[31] Mikki SM, Kishk AA (2009) Electromagnetic scattering by multi-wall carbon nanotubes. Prog Electromagn Res B $17: 49-67$

[32] Thirunavukkuarasu K, Hennrich F, Kamarás K, Kuntscher CA (2010) Infrared spectroscopic studies on unoriented single-walled carbon nanotube films under hydrostatic 
pressure. Phys Rev B 81(4):045424. doi:10.1103/PhysRevB. 81.045424

[33] Hu L, Hecht DS, Grüner G (2009) Infrared transparent carbon nanotube thin films. Appl Phys Lett 94(8):081103. doi:10.1063/1.3075067
[34] Morimoto T, Okazaki T (2015) Optical resonance in farinfrared spectra of multiwalled carbon nanotubes. Appl Phys Express 8(5):055101. doi:10.7567/APEX.8.055101 